\title{
EP-146
}

\section{Early experience and the learning curve for robotic cholecystectomy using da Vinci SP}

\author{
Hyeon Kook LEE*
}

Department of Surgery, Ewha Womans University Seoul Hospital, Seoul, Korea

Introduction: The cholecystectomy by da Vinci Xi is gradually increasing and this operation is proven to be a safe and effective operation. Furthermore single port robotic cholecystectomy (SIRC) is a newly attempted surgical procedure, and is rarely used worldwide. For this reason, early experience with SIRC with da Vinci SP is needed to be shared and discussed about the early results.

Methods: This study is single center retrospective cohort study, total 72 patients underwent SIRC with benign gallbladder disease between 2019 and 2020. Da Vinci SP is used with single multi-channel port though the $2.5 \mathrm{~cm}$ sized umbilical skin incision, cholecystectomy is done using some SP devices like cadiere, Maryland forceps. Patient's demographics, operation time, and postoperative factors were analyzed in each 10 cases.

Results: There was no intra operative injury, early postoperative complication and un-expected hospital visit. The operation time involving preparation time and console time was gradually decreased, every 10 cases showed 37.5, 34.14, 35.9, 29.2, 28.4, 24.83, 20.12 in console time. Compared to the first 30 cases, the last 10 cases showed a significantly shorter time. Demographics, the estimated blood loss and postoperative factors like pain score, hospitalization showed no significant difference in each group.

Conclusions: SIRC using da Vinci SP showed comparable outcome regarding intra-operative factors and postoperative complication, pain and hospitalization. SIRC using da Vinci SP is a surgery that easily develops, although it requires some training and time for beginners to achieve good results such as shortening the operation time. 